\title{
Scheduling Optimization Method of Single-line Customized Bus
}

\author{
Yunwei Li, Xuedong Yan, Wen Shao \\ Beijing Jiaotong University, Beijing, China.
}

\begin{abstract}
Keywords: Validate the model, single-line customized bus was studied.
\end{abstract}
\begin{abstract}
Customized bus, a new-type traveling mode in China, now has become the first choice for part of city residents due to its quality services. However, customized bus in China is still at the initial stage of exploration. Its route design and vehicle scheduling have not yet formed a unified methodology. Therefore, carrying on planning on customized bus is an important issue which should be considered by traffic researchers. In this article, scheduling method of single-line customized bus was studied. A related model was established to obtain customized bus operation plan of single line. Meanwhile, practical examples were used to validate the models, providing references for the development of customized bus in China.
\end{abstract}

\section{Introduction}

Customized bus belonging to the category of Demand Responsive Transport (DRT) is a kind of auxiliary public transportation service mode, which is between conventional bus and taxi. It aims to provide public transport services for people who have similar traveling demand in time and place. According to the passengers demand submitted online or telephone, customized bus transit designs route lines scientifically and meets passenger demands as much as possible. Direct journey, flexible routes, high level services and other advantages are different from conventional bus [1].

The study history of DRT public transport system has been nearly 50 years. Foreign scholars devoted to the study in DRT system designing, system planning, real - time scheduling model and algorithm, launched a number of cutting-edge research [2,3]. China's customized bus transit is still in the exploratory stage. Its route design and vehicle scheduling have not yet formed a unified methodology $[1,4]$.

The purpose of this study is to arrange vehicle routing and resource allocation problem scientifically and reduce the delay time of passengers and the cost of the enterprise. In this paper, a real-time scheduling model for single-line customized bus transit is established in order to make the system optimal. It not only provides reference and theoretical support for opening and implementation of customized public transport system, but also accelerates the construction of urban customized bus transit system.

\section{Problem Description and Modeling}

The paper establishes a model combining with the dynamic scheduling idea [5] and The theory of foreign DRT planning method [6].

\subsection{Problem Description}

The research object in this paper is one-way traffic corridor service type. The traffic corridor is a fixed line. This paper mainly studies the traveling demand from region to region along the corridor. There are all pickup demands in the starting area and all delivery demands in the ending area. Stations are fixed and arranged in order along the corridor. The passenger submits its traveling generation time, traveling OD and the time window, that is, the time range in which the passenger is expected to be served. The dispatching center carries on the reasonable disposition of vehicle resources, and arranges vehicles to respond to the dynamic demand of passengers in order to make the whole system optimal. 


\subsection{Modeling}

\subsubsection{Model Assumption}

1) The stations and distance between any two points are known. The bus travels along the corridor.

2) Vehicle travels at the same speed throughout the road. Road traffic conditions are good and stable. Congestion problem is not considered.

3) We only consider one type of bus. The capacity of the vehicle is a fixed Value.

4) During the study period, passenger reservation is known;

5) The time window of passengers is hard, that is, not allowed to be violated. There is no vehicle waiting for passengers.

\subsubsection{Variable Definition}

$i_{p}(i=1,2, \cdots, n ; p=1,2)$ : Demand $i$ generated at time $\mathrm{t}(\mathrm{p}=1$ denotes pickup demand; $\mathrm{p}=2$ denotes delivery demand)

$j(j=1,2, \cdots, m)$ : Bus station

$I$ : Set of demand

$k(k \in\{1,2, \cdots, N\})$ : Vehicle $\mathrm{k}$

$N$ : Sum of trips in operation period

$A_{j}^{k}$ : Time when $\mathrm{k}$ arrives at $\mathrm{j}$

$D_{j}^{k}$ : Time when $\mathrm{k}$ leaves $\mathrm{j}$

$B_{j}^{k}$ : Time when $\mathrm{k}$ begin to service at $\mathrm{j}$

$t_{i j}^{k}$ : Direct travel time between $\mathrm{i}$ and $\mathrm{j}$ of $\mathrm{k}$

$\gamma$ : Maximum onboard time coefficient

$S_{j}^{k}$ : Stop time(The time of unit passenger on and off the bus is ts.)

$T^{k}=T_{O}+\sum_{j=1}^{k} t_{1}^{k}$ and $T^{N} \leq T_{D}:$ Departure time of vehicle $\mathrm{k}$

$x_{1}(i, j, k)=\left\{\begin{array}{l}1 \\ 0\end{array}\right.$ Demand $\mathrm{i}$ gets on vehicle $\mathrm{k}$ at station $\mathrm{j}=1 ;$ Otherwise $=0$

$x_{2}(i, j, k)=\left\{\begin{array}{l}1 \\ 0\end{array}\right.$ Demand i gets off vehicle $\mathrm{k}$ at station $\mathrm{j}=1 ;$ Otherwise $=0$

\subsubsection{Single-Line Customized Bus Dynamic Scheduling Model}

\section{1) Objective function}

The model should focus on the following two aspects: A) Minimum the cost of the scheduling process. It Includes passenger onboard time and business operation cost. B) Increase the number of passengers served as much as possible. Minimum cost under the premise of meeting the service level and demand of passengers.

a) Minimum related cost

$$
\min C_{1}=\alpha C_{11}+\beta C_{12}=\alpha \sum_{i} \sum_{j} \sum_{k}\left(A_{j}^{k} x_{2}(i, j, k)-B_{j}^{k} x_{1}(i, j, k)\right) n_{i}+\beta \sum_{k}\left(A_{D}^{k}-A_{O}^{k}\right)
$$

In which

C11 is the onboard time of all passengers called passenger onboard cost.

$\mathrm{C} 12$ is the sum of the traveling time of the vehicle called operating cost.

$\alpha$ and $\beta$ are weight coefficients corresponding to passengers onboard cost and the operating cost. $\alpha$ $+\beta=1$.

b) Maximum number of passengers

$$
\max C_{2}=\sum_{k} \sum_{i} \sum_{j} x_{1}(i, j, k) n_{i}
$$

2) Constraints 


$$
\begin{aligned}
& T_{e}^{i} \leq B_{j}^{k} \leq T_{l}^{i}, \quad \forall i, \text { and }, x_{1}(i, j, k)=1 \\
& \sum_{i} \sum_{j} x_{1}(i, j, k) n_{i} \leq Q_{k}, \forall k \\
& A_{j_{2}}^{k} x_{2}\left(i, j_{2}, k\right)-B_{j_{1}}^{k} x_{1}\left(i, j_{1}, k\right) \leq \gamma t_{j_{1}, j_{2}}, \forall i \in I \\
& \quad A_{D}^{k}-A_{O}^{k} \leq T_{\max }, \forall k \\
& \sum_{j} x_{1}(i, j, k)-\sum_{j} x_{2}(i, j, k)=0 \text { or } 2, \forall k, i \\
& h_{\min } \leq t_{1}^{k} \leq h_{\max }, \forall k \\
& T_{O} \leq \sum_{i=1}^{k} t_{1}^{i} \leq T_{D}, \forall k
\end{aligned}
$$

Constraint (3) indicates that the service time of the vehicle must be within the time window of demand reservation when demand i selects station $\mathrm{j}$ as pickup station. Constraint (4) ensures that the number of passengers per bus does not exceed capacity. Constraint (5) ensures that the passengers get off within the get-off time window, so that passenger onboard time is not too long. Constraint (6) is the limit of maximum traveling time for the route. Constraint (7) ensures each demand pair to be served by the same vehicle. Constraint (8) defines the range of departure intervals. Constraint (9) indicates that departure time is within the specified operation period.

\section{Model Solving Method}

In this paper, the genetic algorithm is used to solve the model. Since the model is multi-objective planning, the fitness function is constructed by using the ratio of time cost and total passenger service number to obtain the minimum value of objective function. To solve the problem, the constraints need to be processed. Once the constraint is violated, a penalty function is imposed to be added to the original objective function. The penalty is a relatively large integer to facilitate scheme screening. In this study, the penalty function mainly includes vehicle capacity penalty, passenger onboard time penalty and vehicle traveling time penalty.

\section{Case Studies}

\subsection{Basic Situation of the Selected Line}

\begin{tabular}{|c|c|c|c|c|}
\hline $\begin{array}{c}\text { Alternate station name } \\
\text { Number }\end{array}$ & $\begin{array}{c}\text { FQHYNM } \\
1\end{array}$ & $\begin{array}{c}\text { JYGLSD } \\
2\end{array}$ & $\begin{array}{l}\text { CPDSJ } \\
3 \\
\end{array}$ & $\begin{array}{c}\text { JKY } \\
4\end{array}$ \\
\hline Alternate station name & $\mathrm{CM}$ & JYWKC & YALB & YAGY \\
\hline Number & 5 & 6 & 7 & 8 \\
\hline Alternate station name & STJYZ & STJY & BQHY & ACEIT \\
\hline Number & 9 & 10 & 11 & 12 \\
\hline Alternate station name & RJYDZ & SDQJHDN & SDWJ & SDSJ \\
\hline Number & 13 & 14 & 15 & 16 \\
\hline
\end{tabular}

In this paper, a typical route of DIDI bus transit served commuters is selected as the research object. The route begins in FQHYNM, and ends in SDSJ, connecting Changping District and Shangdi Information Industry Base. Combining the existing principles of station distribution and customized stations on that route, the station set of the route is determined. The station number is as shown in Table 1. Station $1 \sim 11$ are pickup stations. Station $12 \sim 16$ are delivery stations.

Table 1. Number of bus station

\subsection{Results and Analysis}

According to the selected case, the genetic algorithm is used to solve the model. The values of parameters are as follows. [TO, TD] is $[0,180]$. Qk is $33 . \mathrm{v} 1$ is $1.1 \mathrm{~m} / \mathrm{s} . \gamma$ is 1.5 . Tmax is $90 \mathrm{~min}$. [Tmin, Tmax] is $[10,35] . \mathrm{VC}=30 \mathrm{~km} / \mathrm{h} . \alpha=0.9, \beta=0.1$. Crossing probability is 0.7 and variation probability is 0.2 . 


\section{RESULTS}

When given demand and vehicle number, the optimal departure interval scheme can be obtained. Taking $\mathrm{n}=400$ as an example, the model is solved and compared with the conventional bus scheduling results. The results of the comparison are shown in Table 2. Controlling demand scale and vehicles to be same of one route, the per capita time cost of customized bus is less than that of traditional bus. The customized bus scheduling method has certain optimization effect.

Table 2. Scheduling results comparing with traditional bus

\begin{tabular}{lccc}
\hline Category & Vehicle & Departure interval $(\min )$ & Total time cost / The number of passengers served(min) \\
\hline \multirow{2}{*}{ Conventional } & vehicle=10 & $10,10,10,10,10,10,10,10,10,10$ & 57.9270 \\
bus & vehicle=11 & $10,10,10,10,10,10,10,10,10,10,10$ & 57.5455 \\
& vehicle=12 & $10,10,10,10,10,10,10,10,10,10,10,10$ & 57.4810 \\
\hline \multirow{2}{*}{ Customized bus } & vehicle=10 & $12,10,19,10,10,28,10,10,10,34$ & 55.5414 \\
& vehicle=11 & $13,10,18,10,34,14,11,10,10,28,10$ & 55.2921 \\
& vehicle=12 & $10,10,21,10,28,10,10,11,10,10,28,10$ & 55.5218
\end{tabular}

\subsubsection{Impact of Demand Scale and Vehicle Number on Objective Function Values and} Operation Scheme

We explored the relationship between the size of the demand or number of vehicles and the values of objective function by calculating objective function values while changing the other two factors. The results are shown in Table 3.

Table 3. Values of objective function of different schemes

\begin{tabular}{ccccc}
\hline Demand & Vehicle & Departure interval(min) & Time cost & The number of passengers served \\
\hline \multirow{2}{*}{$\mathrm{n}=350$} & vehicle=9 & $10,31,26,10,31,10,34,12,13$ & 8745.1 & 159 \\
& vehicle=10 & $12,10,10,35,10,31,10,34,10,12$ & 9375 & 172 \\
& vehicle=11 & $11,31,10,20,10,27,10,24,10,10,17$ & 10728 & 207 \\
$\mathrm{n}=400$ & vehicle=10 & $12,10,19,10,10,28,10,10,10,34$ & 11663 & 228 \\
& vehicle=11 & $13,10,18,10,34,14,11,10,10,28,10$ & 12607 & 252 \\
\hline
\end{tabular}

From table 3-3, the following results can be gained:

1) When demand is determined, time cost increases roughly with the increasing of the number of vehicles. This is also the same as empirical cognition.

2) When the number of vehicles is determined, time cost is roughly proportional to demand scale.

3) The number of passengers served and time cost also have the same trend as mentioned above. The number of passengers served is proportional to the demand (or supply) when the supply (or demand) is constant while the number of passengers onboard does not exceed the total capacity of the vehicle.

4) Different departure intervals are obtained depending on different combination of demand scale and vehicle number. The model can provide the optimal departure intervals according to different supply and demand and obtain a relatively optimized operation scheme, providing reasonable reference for enterprise decision-making.

\subsubsection{Space-Time Analysis of Customized Bus}

Figure 1 is the space-time diagram of delivery stations when vehicles' number is 8 and demands' is 300 . The chart clearly shows passenger serving states, operation and scheduling states of vehicles at any station, provide reference for decision-making of enterprises. 


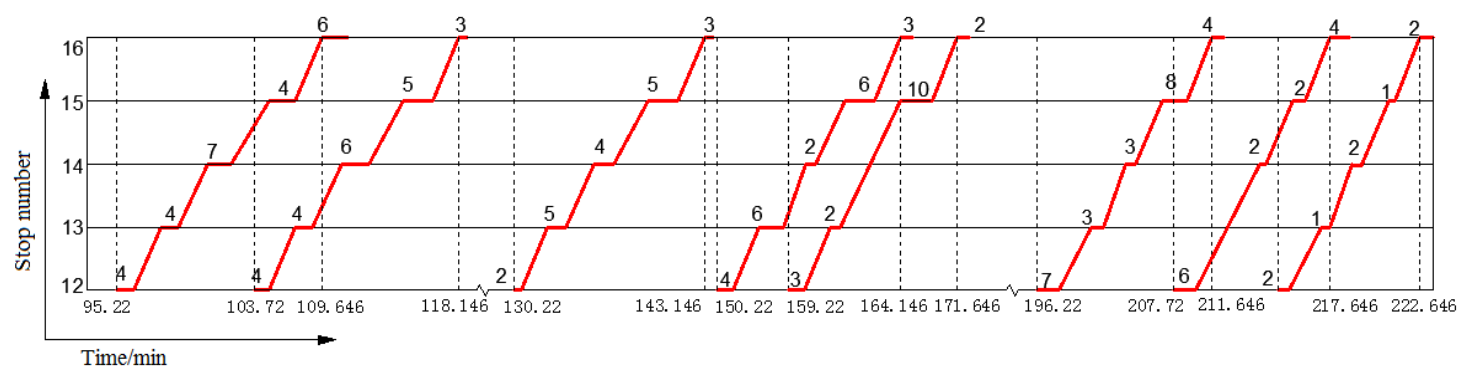

Figure 1. Space-time diagram of delivery stations

\section{Conclusion}

In this article, scheduling method of single-line customized bus was studied. The model was established using dynamic dispatching idea. The main goals of the model were to minimize the passenger travel costs and transit operating costs. In the end, this paper obtains the operation plan and analyzes the impact of the demand scale and the number of vehicles on the cost. Case study shows that the model can provide enterprises with more optimized decision-making combination, reasonably arranges the vehicle resources and coordinates the departure time to ensure that passenger demands are met. The real-time scheduling method proposed in this paper effectively considers the shortcomings that our current customized transit system cannot make an immediate reservation. To a certain extent, it provides a corresponding methodology support for China's customized bus operation.

\section{References}

[1]. HU Liang, ZHANG Wenjie. Brief Analysis of Customized Bus[J]. Highways \& Transportation in Inner Mongolia, 2014, 02: 72-74. (in Chinese)

[2]. Schofer JL. Resource Requirements for Demand-responsive Transportation Services[M]. USA: National Reader Press, 2003.

[3]. Ghiani G, Guerriero F, Laporte G, Musmanno R. Real Time Vehiele Rougting Solution Concepts, Algorithms and Parallel Computing Strategies[J]. European Journal of Operational Research, 2003, 151(1): 1-1.

[4]. LIU Dongmei. The Transit Scheme Research on Customized City Bus Service of Xi'an[D]. Chang'an University, 2014. (in Chinese)

[5]. WU Lirong. Areal-time Scheduling Model for Demand Responsive Campus Bus System[D]. Dalian University of Teehnology, 2011. (in Chinese)

[6]. Paquette J, Cordeau J F, Laporte G, et al. Combining Multicriteria Analysis and Tabu Search for Dial-a-ride Problems[J]. Transportation Research Part B: Methodological, 2013, 52: 1-16. 